\title{
Démocratie et Etat de non-droit au Brésil : analyse et témoignage
}

Paulo Sérgio Pinheiro

\section{(2) OpenEdition \\ Journals}

Édition électronique

URL : http://journals.openedition.org/conflits/1887

DOI : $10.4000 /$ conflits. 1887

ISSN : $1777-5345$

Éditeur :

CCLS - Centre d'études sur les conflits lilberté et sécurité, L'Harmattan

Édition imprimée

Date de publication : 1 septembre 2005

Pagination : 87-115

ISBN : 2-7475-9476-9

ISSN : 1157-996X

Référence électronique

Paulo Sérgio Pinheiro, «Démocratie et Etat de non-droit au Brésil : analyse et témoignage », Cultures \& Conflits [En ligne], 59 | automne 2005, mis en ligne le 01 octobre 2005, consulté le 30 mars 2021. URL http://journals.openedition.org/conflits/1887 ; DOI : https://doi.org/10.4000/conflits.1887

Ce document a été généré automatiquement le 30 mars 2021.

Creative Commons License 


\title{
Démocratie et Etat de non-droit au Brésil : analyse et témoignage
}

\author{
Paulo Sérgio Pinheiro
}

1 Il existe, dans les nouvelles démocraties d'Amérique latine, un écart entre la loi et sa mise en œuvre effective. Sur ce continent, l'égalité de tous les citoyens devant la loi ne correspond nulle part complètement à la vie réelle. Toute intervention de l'Etat se fait au nom de la communauté tout entière - sans que cessent pour autant les transgressions de la loi par ceux qui sont censés protéger les intérêts de la collectivité mais ne sert très souvent qu'à renforcer le pouvoir des élites.

2 On peut observer après la fin de la monarchie au Brésil le 15 novembre 1889 quatre périodes constitutionnelles issues de quatre transitions politiques. La première suit la proclamation de la République en 1889 et la Constitution de 1891 et se poursuit jusqu'à la « révolution de 1930 » qui a «destitué » le Président Washington Luiz (1870-1975, président entre 1926-1930) ; la deuxième, après la Constitution de 1934 ouvre le plus court interrègne démocratique de notre histoire, jusqu'en 1937 quand un coup d'état militaire sous le leadership de Getulio Vargas (1930-1954) ${ }^{1}$ institue, avec l'appui de l'armée, la dictature d'inspiration fasciste de l'« Estado Novo » qui durera jusqu'en 1945 quand Vargas est destitué par l'armée ; la troisième, celle ouverte par la promulgation de la Constitution de 1946 va durer jusqu'au coup d'état militaire de 1964 et verra une succession de gouvernements dictatoriaux jusqu'à 1985 ; la quatrième, après la fin de la dictature militaire, le retour du gouvernement civil en 1985 et la constitution «citoyenne» de 1988. On pourrait espérer, en théorie, que les constitutions démocratiques établissent les conditions de l'exercice de la violence physique par l'Etat et assurent les garanties des citoyens devant l'arbitre.

3 Partant, aucune des déclarations de droits ${ }^{2}$ figurant dans les constitutions démocratiques ou dictatoriales n'a jamais été respectée. L'article 72 de la première constitution républicaine de 1891 présentait une longue et solennelle énumération des droits et garanties - pas très différents, ni de moindre importance de ceux figurant dans les constitutions de 1934, 1937, 1946 (ou dans la constitution dictatoriale de 1967, 
ou dans l'amendement $n^{\circ} 1$ de 1969), et qui n'ont été dépassés en précision que par la Constitution de 1988, et n'ont jamais été suivis d'effets.

4 Depuis la fin du $19^{\text {ème }}$ siècle et tout au long du XX ${ }^{\text {ème }}$ siècle le Brésil a connu de grands changements comprenant la modernisation de l'Etat, des transformations formidables de l'économie, la complexification de la vie sociale, le passage d'un pays rural à urbain et le renforcement des organisations de la société civile. On ne peut nier que dans la période républicaine suivant 1889 de grandes transformations sont intervenues en ce qui concerne les classes dominantes et les classes populaires, leur composition, leurs motivations, leurs projets et leurs pratiques. Lors de la première République, une classe ouvrière naît, formée par une main-d'œuvre étrangère, immigrée ; entre 1930 et 1937, dans une conjoncture marquée par l'avènement du fascisme, et après 1946, au moment de la Guerre Froide, les classes dirigeantes craignent le "péril rouge " et s'attaquent aux communistes et à leurs alliés. Sous le populisme, la répression à l'encontre du mouvement ouvrier dure jusqu'en 1950, quand Getúlio Vargas est élu Président de la République par le vote des travailleurs. Pendant un temps, les autorités maintiennent les interventions dans les syndicats; ces interventions diminuent peu à peu et les grèves recommencent ${ }^{3}$. Lors des gouvernements suivants, la répression "politique " devient moins intense ou plus limitée, selon la conjoncture politique : par exemple, au moment de la crise survenue le 25 août 1961, lorsque le président Jânio Quadros (1917-1992, président de janvier à août 1961) a donné sa démission, la police politique, profitant de la situation, reprend la persécution à l'encontre des communistes ${ }^{4}$. Cependant, l'arbitraire policier à l'égard des personnes suspectées de crimes de droit commun semble ne jamais s'interrompre. Après 1985, l'Etat fait face à des mobilisations de la société civile qui se développent à une échelle beaucoup plus importante qu'à n'importe quel autre moment par le passé, de même qu'il fait face à une violence criminelle endémique.

5 Le cercle vicieux des "verticalités des inégalités brésiliennes » a toujours favorisé la conciliation et les transitions pactisées, sans ruptures avec le passé qui, à leur tour, collaborent à leur façon au maintient de ces mêmes verticalités ${ }^{5}$. Les gouvernements sous le constitutionnalisme démocratique - auquel on ne peut pas inclure la Vieille République de 1889-1930 au caractère franchement oligarchique et autoritaire malgré une Constitution issue d'une assemblée élue - comprennent le bref interrègne de 1934-1937, le régime de la constitution de 1946 jusqu'au coup d'état de 1964 et le régime de la constitution de 1988 jusqu'à présent. Ils n'ont jamais été capables de contrôler effectivement les pratiques arbitraires des agents de l'Etat. Il s'agit, bien sûr, dans des conjonctures bien différentes, de phénomènes essentiellement distincts lorsqu'on parle de la répression politique sous la dictature, d'une part, et des formes arbitraires de répression du crime d'autre part, ainsi que de la répression généralisée des classes populaires sous la démocratie.

6 Il me semble que pour comprendre les places respectives de l'autoritarisme et de la démocratie au Brésil dans les vingt dernières années on doit tenir compte de ce lourd héritage dont on indique à peine ici les contours fondamentaux. Même dans des moments fondateurs de la démocratisation, comme en 1985, les vestiges de l'autoritarisme persistent, et les mouvements d'opposition, lorsqu'ils arrivent au gouvernement, ne parviennent pas à mettre en place des réformes susceptibles de remettre en cause la nature autoritaire du gouvernement et de la société. A la lumière de ce passé, je discute ici dans la décennie des années 1990 jusqu'à présent les questions 
relatives aux garanties constitutionnelles, en particulier les droits civils, et le fonctionnement du pouvoir judiciaire et de la police, en essayant d'attirer l'attention sur la violence endémique et les violations systématiques des droits humains sous les gouvernements constitutionnels démocratiques, en particulier dans les années 1990. J'y examine les efforts déployés au Brésil par le gouvernement et la société civile en vue d'un élargissement de la jouissance des droits de la personne à toute la population. Enfin, cet essai comporte également mon témoignage sur mon bref passage au gouvernement fédéral du Brésil.

\section{Une citoyenneté très limitée}

7 Même si les garanties fondamentales du citoyen ont toujours été bien définies dans les Constitutions brésiliennes, comme nous l'avons déjà indiqué, l'exercice d'une pleine citoyenneté est toujours resté limité pour le plus grand nombre. De tous temps, dès l'Antiquité pourrions nous dire, une Constitution trouve ses racines dans le système social ${ }^{6}$. Et comment cela pourrait-il en être autrement pour le Brésil ? Il faut bien se rendre compte que loin des idéaux qui figurent dans les Constitutions, les procédures juridiques et le fonctionnement de la loi reflètent les réalités cruelles de la société brésilienne. Ils n'arrivent pas à atténuer les énormes différences entre les pauvres et les riches $^{7}$ : le système juridique est un outil et un réflexe de la société et, donc, de l'inégalité sociale. Il ne se place pas en dehors et au-dessus de la société et des réalités sociales, comme ayant une essence propre, une logique autonome ou une existence indépendante. L'Etat ne peut pas être différent de la société elle-même : il est le lieu où s'affrontent des intérêts, des conflits de classes. Dans ce cadre, tout gouvernement dépend de l'organisation particulière d'une société : un gouvernement, comme c'est le cas du Brésil, ne peut pas opérer sur des bases démocratiques dans une société où, à différentes périodes constitutionnelles, les femmes et les analphabètes ne votent pas, où les travailleurs ruraux et les domestiques ne sont pas défendus par des normes sociales, où une colossale disparité subsiste entre les revenus des citoyens, et surtout où ce tout social s'articule à un racisme structurel contre les Brésiliens d'origine africaine qui constituent $46 \%$ de la population ${ }^{8}$.

8 Aucun régime ne peut être vraiment démocratique si ceux qui n'appartiennent pas à l'élite n'ont aucun accès aux droits ni aux instruments assurant un contrôle social effectif des élites. En plus des exigences minimales d'un constitutionnalisme démocratique - liberté de réunion, liberté d'expression, suffrage universel, élections régulières et propres, l'indépendance des pouvoirs - la démocratie réclame la réalisation d'un ensemble toujours plus élargi d'exigences. Celles-ci constituent l'état de droit, le due process of law, le droit à un jugement juste et équitable, au respect de l'intégrité physique des citoyens. Pendant toute la période républicaine au Brésil, quel que soit le régime, dictatures et démocraties confondues, on a toujours répondu de façon très restreinte à ces demandes.

9 Après chaque transition politique, aucune des nouvelles classes dirigeantes n'a rendu effectives les grandes aspirations en matière de libertés et de droits qui ont marqué les mouvements de résistance. La criminalisation de la dissidence et des mouvements populaires dans les périodes non dictatoriales est une constante qui se manifeste à travers le temps (même s'il ne s'agit pas là d'un exotisme brésilien) : le travailleur immigrant comme révolutionnaire, les anarcho-syndicalistes entre 1900-1920, les 
communistes dans les années 1930 ou 1945, les syndicats urbains et ruraux dans les années 1960, les travailleurs sans terre dans les années 1990 et 2000 ont toujours été définis comme des délinquants sociaux, comme l'a si bien montré Michael Hall9. Toute mobilisation populaire, ouvrière et paysanne (faut-il rappeler que les Ligues Paysannes de Francisco Julião, dans le Nord-est, ont été fortement réprimées avant le coup d'état militaire de 1964 ?), toute "violence défensive", selon l'expression de Herbert Marcuse $^{10}$, tend à être empêchée par les agents de l'Etat, grâce à l'usage récurrent d'une violence illégale, bien plus importante que le recours à l'extra légalité "normale", inhérente à la forme étatique. Rien ne montre aussi bien les limitations chroniques de l'Etat démocratique que le cas du Brésil entre 1946 et 1964, et après 1988 : un Etat est ce que le gouvernement en fait ${ }^{11}$.

10 Malgré les modifications qui ont bouleversé la société brésilienne, chaque nouvelle étape politique non autoritaire a été marquée par la permanence accumulée d'éléments hérités de l'esclavage, de la concentration de la propriété de la terre et des revenus, de l'inégalité sociale et raciale, des micro-despotismes (selon l'expression de Guillermo o'Donnell) et d'un système complexe - très coriace et en même temps subtil - de hiérarchies ${ }^{12}$. Les pratiques autoritaires, plus visibles dans les institutions de contrôle de la violence et du crime, comme la justice et la police, furent très peu modifiées après les changements de régime politique ou l'avènement d'élections démocratiques.

11 On ne prétend pas faire croire que le passé se prolonge automatiquement dans le futur grâce à un déterminisme quelconque d'ordre économique, psychologique ou sociologique. Nous ne voulons pas prétendre que les facteurs explicatifs de la violence et des violations des droits sont restés immuables au fil de toute la période républicaine. Pour une raison bien simple, comme l'a remarqué Michel Debrun, parce que "dans la même société, la force originaire de chaque passé se heurte à la force originaire d'autres passés, mais parce que les tendances - sauf quand elles sont cristallisées, et alors ce ne sont pas exactement des tendances mais des habitudes, des routines - comprennent toujours une composante de faiblesse $»^{13}$. Les continuités n'empêchent donc pas que des configurations nouvelles et inattendues puissent émerger à chaque conjoncture, telles des figures kaléidoscopiques, composées par des réseaux d'interdépendances entre des groupes et des acteurs politiques et sociaux ${ }^{14}$.

\section{Les stratégies de la conciliation}

12 Les obstacles à la consolidation démocratique persistent car, dans toutes les transitions, face aux risques de rupture des équilibres de pouvoir susceptibles d'être provoqués par la mobilisation populaire qui invariablement les accompagne, les anciennes oppositions se coalisent avec des forces issues de l'establishment antérieur. Ces coalitions, avec des forces politiques conservatrices et très résistantes aux changements annoncés par les nouvelles forces au gouvernement ont été constantes, comme l'a clairement signalé Michel Debrun dans son approche de la conciliation comme véritable « archétype » de l'action politique brésilienne ${ }^{15}$. Il faut dire qu'un « archétype » n'est pas déterminé par son contenu mais seulement par sa forme: l'archétype peut se trouver dans des conjonctures très distinctes ${ }^{16}$. En effet, la " conciliation » ne s'est pas développée dans le but d'éviter des conflits entre des adversaires de force comparable, "mais, au contraire, pour formaliser et réglementer le rapport entre des acteurs inégaux, les uns déjà dominants et d'autres déjà dominés. Et pour permettre que les premiers puissent exploiter à leur 
avantage les seconds en tant que des associés caudataires ». Les transitions servent presque toujours à renforcer ceux qui avaient déjà du pouvoir ${ }^{17}$. Chaque transition reprend donc la forme de l'archétype de la conciliation et chaque époque lui donne des contenus différents.

\section{Le « transformisme » et la continuité} transitions politiques, n'oseront ou ne seront pas capables de se confronter avec l'héritage autoritaire ou ne le voudront pas. Cet héritage a toujours été sous-estimé par les anciennes oppositions lorsque celles-ci arrivent au pouvoir. Formées dans la résistance aux gouvernements autoritaires et au sein des mouvements sociaux pendant les transitions politiques, elles tendent à surestimer leurs propres capacités à promouvoir la démocratie sur des bases volontaristes sans avoir à régler la question du récent passé autoritaire. Dès leur accession au pouvoir, les partis politiques issus des anciennes oppositions ont le plus souvent renoncé à se débarrasser des «restes autoritaires " (entulho autoritário) laissés par le régime autoritaire précédent et à promouvoir les réformes des institutions héritées de la dictature. semblent souffrir d'un "transformisme $»^{18}$ qui voudrait qu'un parti progressiste auparavant dans l'opposition ne se transforme en un parti conservateur. Lorsque les cadres issus du réformisme radical qui a prôné le changement prennent en main les rouages du gouvernement, l'ancien radicalisme ne survit pas. Dans les politiques des nouveaux gouvernements, après la transition, il est difficile de trouver des traces des agitations de rues, des insurrections, des réunions de dissidents. La nature des groupes qui se présentaient comme des extrémistes quand ils étaient dans l'opposition aux régimes d'exception ou à la dictature se modifie.

Cette incapacité des classes dirigeantes à transformer l'ordre hérité des régimes autoritaires précédents a des conséquences : les institutions non réformées n'arrivent pas à contrôler les formes d'incivilité présentes dans la société brésilienne ${ }_{20}^{0}$ toujours plus graves à la suite des régimes d'exception 㡠 et dans le cadre démocratique. La succession de chaque période autoritaire aggrave et réactive l'héritage autoritaire.

Evidemment, nous sommes conscients du fait que toutes les formes de société civile sont ravagées par des sources endogènes d'incivilité et que c'est là une caractéristique chronique de la société ${ }^{19}$. Au Brésil, les formes de répression arbitraire développées par l'Etat (ou les classes dominantes) pendant les périodes dictatoriales ont tendance à persister après cette phase, en pleine période d'organisation démocratique. Cette violence physique ouverte, interrompue contre les dissidences politiques, continue à déferler sur les populations pauvres et misérables. L'immense majorité des personnes punies ou arrêtées, à l'exception de ceux qui pratiquent des homicides ou des crimes hideux, est formée de pauvres dépourvus de moyens pour payer un avocat : au Brésil comme partout ailleurs en Amérique Latine, l'application du droit pénal est marquée par son caractère éminemment discriminatoire ${ }^{20}$.

Cependant, les limites de la démocratisation ne sont pas imposées par un personnel politique pratiquant ce «transformisme ». Bien au-delà de ce qui se passe sur la scène politique elle-même, nous devons considérer qu'un autre processus est à l'œuvre au 
sein même des appareils d'Etat. Il peut être très clairement observé au cours des deux décennies qui ont suivi la fin de la dictature militaire en 1985. Il part de l'idée selon laquelle il faut abandonner l'illusion présente lors de la résistance antérieure à la transition politique et consiste à percevoir l'appareil d'Etat comme quelque chose de neutre, capable de s'accommoder de façon automatique à la démocratie. Peut-être fautil justement se rappeler que «les relations qui constituent le pouvoir d'Etat imprègnent la structure même de son appareil, celui-ci étant la condensation d'une relation de forces. C'est précisément cette nature de l'Etat - conçu comme relation - traversé d'un bout à l'autre par des contradictions de classes, qui attribue et légitime un rôle déterminé, et attribue un poids spécifique aux appareils et aux agents qui le composent " ${ }^{21}$.

Lors de la transition de 1985, il n'y a pas eu d'altération des positions des classes dominantes : la transition n'a été possible que grâce à la défection de quelques leaders ou soutiens partisans importants de la dictature militaire. On ne pouvait donc pas s'attendre à des changements dans la constitution même des appareils d'Etat. L'amnistie «ample, générale et sans restrictions », selon la formule du gouvernement militaire, a contribué à les renforcer et à en cristalliser les pratiques. Aujourd'hui, quand nous mesurons la difficulté à promouvoir des reformes, comme par exemple celle de la police, nous nous rendons compte du rôle déterminant et du poids spécifique des institutions, et des appareils qui résistent aux transformations ${ }^{22}$.

$19 \mathrm{Au}$ "transformisme " politique correspond donc une impossibilité de transformation réelle des appareils coercitifs de l'Etat. Tous ou presque se modernisent, comme ceux liés à la gestion économique ou bancaire par exemple; mais la police ou la justice continuent à fonctionner sur une autre temporalité, malgré la démocratie. Dans la période comprise entre 1985 et 2003, parmi les 1300 homicides liés aux conflits agraires, seuls 75 ont été jugés. ${ }^{23}$ Ce dysfonctionnement persistant de l'Etat en matière de justice pénale s'explique par la permanence de ce qu'on pourrait désigner comme « un réseau parallèle » au sein de l'Etat qui survit à tous les changements de régime, qu'il s'agisse des dictatures ou des périodes démocratiques. Réseau, car il se déploie transversalement sur plusieurs secteurs dans différentes branches de l'appareil d'Etat. Parallèle - parce que derrière la façade de fonctionnement légal des organes d'Etat on retrouve un fonctionnement dissimulé, faisant usage de la violence illégale (comme celui de la torture) ou dominé par la corruption ${ }^{24}$. La nature étatique de ce réseau vient de ce qu'il est une ressource à l'intérieur de l'appareil d'Etat, toujours susceptible d'être mobilisée par les classes dirigeantes.

Les racines de ce phénomène ne se trouvent pas dans un mauvais fonctionnement des organes coercitifs d'Etat. Ce réseau n'est pas accidentel ou exceptionnel : sa longévité démontre qu'il est très fonctionnel du point de vue de la domination exercée par les élites par la reproduction de la pauvreté, la concentration de revenus et l'exclusion de la majorité de la population de l'accès au droits les plus fondamentaux, malgré les acquis évidents des successives élections démocratiques qui ont ouvert la voie au parlement et même au gouvernement de représentants des classes sociales traditionnellement exclues. L'existence de ce réseau a très clairement été perçue par Fernando Henrique Cardoso $^{25}$ pendant la dictature militaire, lorsqu'il parlait des « anneaux bureaucratiques » comme expression de la collusion, à l'intérieur de l'Etat, entre les grands entrepreneurs et les échelons élevés de la bureaucratie, pour définir quand et vers quels secteurs devaient s'orienter les investissements publics. On peut encore évoquer d'autres articulations horizontales qui ont eu cours pendant la 
dictature militaire entre différents services de renseignement au sein des forces armées, la police et quelques groupes d'entrepreneurs, par exemple ceux qui assuraient de leur appui financier l'« Opération Bandeirantes » crée par le gouvernement de l'Etat de São Paulo, chargée de la répression clandestine des groupes dissidents. Actuellement, on pourrait évoquer des « anneaux bureaucratiques » d'un type nouveau, articulant entre eux des groupes au sein des services judiciaires, des membres de la " classe politique ", des secteurs des polices, et les services de renseignement dans des pratiques de corruption ou dans le crime organisé, comme l'ont bien démontré plusieurs opérations effectuées par la police fédérale au cours de l'année 2004. De même qu'au temps de la dictature ces "anneaux" fonctionnaient pour assurer une répression en parallèle, aujourd'hui certains d'entre-eux gardent des liens, par le biais de la corruption ou de façon plus directe, par le blanchiment d'argent et le trafic de drogues.

\section{Les paradoxes de la démocratie après les années 1990}

Les réalisations de l'Etat brésilien au cours du $\mathrm{XX}^{\text {ème }}$ siècle ont été remarquables à maints égards: une société fondée sur une économie agraire à peine sortie de l'esclavage est devenue la quinzième (il y a deux ans, la huitième) économie industrielle du monde. L'Etat a montré une efficacité extraordinaire dans l'appropriation des ressources du pays pour promouvoir sa transformation. Cependant, ces ressources se sont concentrées sur des groupes privilégiés qui se sont succédés au long du siècle : nous sommes en présence d'une "gigantesque machine à fabriquer de la pauvreté » (l'expression est de l'écrivain Arundhati Roy ${ }^{26}$ à propos de l'Etat indien) et à concentrer des richesses, en jetant des miettes aux pauvres.

Dans les vingt dernières années du XX ${ }^{\text {ème }}$ siècle, malgré la transition politique de 1985 et le fait pour le Brésil d'être devenu un pays différent de celui du temps de la dictature militaire, son modèle de distribution des revenus reste l'un des plus iniques au monde. Le Brésil est le pays d'Amérique Latine dont la distribution des revenus est la plus inégalitaire. Elle y revêt une extraordinaire stabilité, mis à part un déclin rapide observable dans la période 1989-1992, lors du "plan Cruzado ». Quant au «plan Real », mis en place à partir de 1994, malgré le contrôle de l'inflation et la réduction des niveaux de pauvreté extrême qui en a résulté, il n'est pas évident qu'il ait eu un impact significatif sur la réduction des inégalités qui ont atteint, en 1998, le niveau le plus élevé des deux décennies précédentes ${ }^{27}$. Il faut reconnaître que l'extrême pauvreté (des revenus équivalents à un quart du salaire minimum) a été réduite: en 1992, son contingent représentait $19 \%$ de la population et en $2003,14,5 \%$, soit une réduction de $25 \%$. Cependant, dans les régions métropolitaines, les chiffres sont restés stables comme à Rio de Janeiro - ou ont augmenté : à São Paulo ce contingent s'est élevé de $16 \%$, les habitants en situation de pauvreté extrême passant à cette période de $8,5 \%$ à $9,9 \%{ }^{28}$

Mais on ne peut pas dire, comme le rappelle Lúcio Kowarick, qu'il y ait «déficit de démocratie dans le système politique brésilien $»^{29}$ : on comptait 7,4 millions d'électeurs aux élections de 1945, lesquels correspondaient à $16 \%$ de la population; ils étaient plus de cent millions aux municipales de 1996, soit deux tiers de la population. L'avènement du suffrage universel, un système électoral soumis au judiciaire et fonctionnant d'une manière autonome de l'exécutif, a ouvert l'accès au pouvoir aux classes moyennes et 
aux partis progressistes (comme le PSDB, le parti social démocrate brésilien de Cardoso et le parti des travailleurs de Lula). Toutefois, malgré cette performance remarquable, la démocratie politique reste marquée par un héritage autoritaire perceptible dans les pratiques des gouvernements et des agents de l'Etat et par la discrimination raciale (malgré des progrès évidents comme la criminalisation du racisme après la constitution de 1988 et après 1994 des initiatives de politiques affirmatives en faveur des descendants des africains). Au lieu d'une consolidation démocratique inscrite dans la perspective de la protection des droits de l'Homme, on risque au Brésil de ne promouvoir qu'une consolidation de la démocratie sans citoyenneté. Plus d'un siècle après la proclamation de la République, la rupture avec les continuités reste à faire.

\section{La démocratie et la violence illégale de l'Etat}

L'avènement de la démocratie n'a pas suffi à garantir le contrôle de la violence illégale de l'Etat, même si cette violence n'est pas soutenue par le gouvernement fédéral - bien que, quelquefois, ces crimes bénéficient de la tolérance, voire même de la complicité de certaines autorités - ni à maitriser la violence endémique dont pâtit la société. Les autorités fédérales ne sont pas sans ignorer les graves violations des droits de l'Homme pratiquées par les agents de l'Etat, comme cela a été le cas sous la première république, celle des oligarchies (1889-1930), après la transition de 1945 ou pendant les gouvernements de la démocratie populiste jusqu'au coup d'Etat de 1964. Mais, d'autre part, les gouvernements démocratiques n'ont pas été capables non plus de faire face à une violence endémique marquée par la spirale des homicides, de la criminalité et de conflits agraires qui chaque année ne cessent de s'aggraver.

$\mathrm{Au}$ cours des vingt dernières années (alors que la population est passée de 119 millions d'habitants en 1980 à 170 millions en 2000, ce qui équivaut à une croissance de $43 \%$ ), 598367 Brésiliens ont été assassinés. Dans cette même période, le taux de mortalité par homicide dans le pays a connu un accroissement de $130 \%$, passant de 11,7 à 27 morts pour 100000 habitants. Environ $62 \%$ de ces homicides ont eu lieu dans les années 1990 : en 1997, il y a eu au Brésil plus de 40000 homicides pour une population de 156 millions d'habitants. Le Brésil présente un taux de 27,1 homicides pour 100000 habitants, ce qui le situe au quatrième rang mondial, immédiatement après la Colombie (avec 68), El Salvador $(37)$ et la Russie $(28,4)$. Ces chiffres sont très supérieurs à ceux qu'on observe dans d'autres économies industrialisées : les Etats-Unis d'Amérique, le pays le plus violent du Groupe des Sept (G7), présente aujourd'hui un taux d'homicide de 11 pour 100000 habitants. Si l'on considère la tranche d'âge des jeunes Brésiliens entre 15 et 24 ans, ce taux d'homicide double et atteint 54,1 pour 100000 habitants. Pire encore, la croissance des homicides à l'encontre des jeunes est plus importante que la croissance du taux d'homicide dans la population en général ${ }^{30}$.

Dans les conflits agraires, bien que les chiffres des homicides soient inférieurs à ceux qu'on observe dans les villes (à Rio de Janeiro et à São Paulo la moyenne annuelle d'homicides en 2002 a été d'environ 700), le nombre de travailleurs agraires assassinés a augmenté : en 2003, 73 travailleurs ont été assassinés lors de ces conflits, soit une augmentation de 69,8\% par rapport à l'année précédente. Au total, 1690 conflits agraires ont été enregistrés en $2003^{31}$.

En milieu urbain, le taux d'homicide et le nombre élevé des victimes de cette violence sociale se concentrent largement au sein de la majorité pauvre et misérable, dont les 
descendants des Africains, qui dans leur ensemble représentent la catégorie sociale la plus démunie. Les victimes noirs et métisses constituent des cibles sociales privilégiées : le taux d'homicides de blancs est de 20,6 pour 100000 habitants, et de noirs de $34 \%$ pour 100000 habitants. La proportion de victimes d'homicides dans la population noire ou métisse est donc de $65,3 \%$ supérieure à celle parmi les blancs ${ }^{32}$. Dans ce cadre, il faut rappeler que les travailleurs blancs brésiliens possèdent en moyenne des revenus deux fois plus élevés que les travailleurs noirs (negros) et métis (pardos), selon une recherche effectuée dans six régions métropolitaines du pays. Le revenu moyen des travailleurs blancs était de $\mathrm{R} \$ 1096$, un montant équivalent à $205 \%$ de celui imparti aux travailleurs noirs et métis, $R \$ 535$. Les différences entre noirs et blancs sont encore plus importantes, selon les données de la « Recherche Mensuelle de l'Emploi » (Pesquisa Mensal de Emprego, PME), qui compare le profil de l'emploi entre ces deux groupes. Les noirs et métis sont beaucoup plus présents dans des emplois à faible niveau de qualification qui procurent donc les salaires les plus bas. Parmi la population noire ou métisse occupée en mars de 2004, 11 \% étaient employés comme domestiques et $10 \%$ dans le bâtiment. Parmi les blancs, ces pourcentages étaient respectivement de $5 \%$ et $6 \%^{33}$. Ces données montrent clairement la persistance d'un racisme structurel auquel la démocratie n'a jamais cherché à répondre d'une manière effective.

Une des « institutions de la violence », pour utiliser l'expression de Franco Basaglia, la torture, continue à être systématiquement utilisée par la police civile à l'encontre des suspects pauvres, très souvent des non-blancs, dans tous les commissariats de police du pays $^{34}$. Un rapport récent analysant les recommandations effectuées pendant la mission de l'ancien rapporteur sur la torture des Nations Unies, Sir Nigel Rodley, lors de sa visite au Brésil en août et septembre 2000, a signalé les faiblesses persistantes en matière de politiques publiques censées combattre la pratique systématique de la torture dans le pays ${ }^{35}$. Tous les gouvernements depuis 1985 ont condamné la torture, la convention contre celle-ci fut ratifiée, mais les gouvernements démocratiques ont été incapables de mettre fin à la torture appliquée aux criminels de droit commun. Entre octobre 2001 et le 31 juillet 2003, SOS Torture, un service téléphonique mis en place pour recevoir des dénonciations anonymes de cas de torture, a enregistré 1336 cas de torture institutionnelle. La torture est pratiquée dans tous les Etats de la fédération, même les plus développés. Minas Gerais, São Paulo et Pará ont enregistré le plus grand nombre de cas. Presque la moitié des faits indiqués concernent des commissariats de police. 778 d'entre eux ont été saisis par le Parquet, mais 31 cas seulement ont fait l'objet de poursuites devant les tribunaux, avec six condamnations et quatre acquittements ${ }^{36}$.

Désormais, les « indésirables » ne sont plus «bannis » pour l'Amazonie ou expulsés du pays, comme c'était le cas sous la vieille République jusqu'en 1930 : très souvent on les tue. Les exécutions sommaires par la police à São Paulo et Rio de Janeiro sont non seulement tolérées, mais dans certains cas aussi stimulées par les autorités. 264 personnes ont été tuées par des policiers en service ou en congé à São Paulo au cours du premier trimestre de l'année 2003, faisant presque trois morts par jour. Ce chiffre est supérieur au nombre de personnes tuées par des policiers au cours des dix dernières années $^{37}$. Les chiffres sont très similaires à Rio de Janeiro; et même si dans d'autres Etats les morts sont moins nombreuses, la tendance reste la même. Une comparaison entre le Brésil et d'autres pays démocratiques (hormis ceux qui vivent des situations de guerre insurrectionnelle comme la Colombie), permet de vérifier que c'est au Brésil que 
l'on trouve le taux de violence policière létale le plus élevé au monde au cours des vingt dernières années.

Les efforts du ministère public pour mettre en accusation de nombreux policiers militaires impliqués dans des assassinats et des massacres, même si quelques condamnations ont été obtenues, n'ont pas abouti dans un bon nombre de cas. Ces échecs sont souvent dus au fait que les enquêtes concernant les crimes commis par des policiers militaires sont effectuées par la police militaire elle-même 臨 il s'agit d'un IPM : Inquérito Policial Militar, réalisé de façon non professionnelle, sur la base de preuves réunies de façon très superficielle. C'est ce qui s'est passé en 1995, à Corumbiara, Etat de Rondônia, à l'occasion d'un massacre dont ont été victimes douze paysans sans terre: parmi les quatorze policiers militaires accusés, neuf ont été acquittés à la suite d'un jugement rendu entre août et septembre 2000. L'impunité des agents de l'Etat est virtuellement assurée pour ceux qui pratiquent des crimes à l'encontre de victimes pauvres, considérées « indésirables » ou « sous humaines ».

31 En ce début de siècle, un grand nombre d'institutions, relevant de la compétence des gouvernements des Etats, plutôt que de sauvegarder l'Etat de droit, continuent à le rogner en utilisant des tactiques brutales et létales pour traiter la violence. Il en est ainsi des actes de torture systématiques pratiqués, entre octobre 1999 et septembre 2000, à l'encontre d'adolescents délinquants enfermés à la FEBEM (Fundação do Bem Estar do Menor, Fondation du bien être de l'enfant, gouvernement de l'Etat de São Paulo) à São Paulo, y compris à l'encontre de ceux qui avaient dénoncé ces pratiques; il en est également ainsi des assassinats de militants du mouvement des (paysans) sans terre lors des massacres commis par des policiers militaires ou par des employés d'entreprises de sécurité privées payés par des propriétaires de domaines (fazendeiros), comme au Mato Grosso do Sul en 2000. Pour les policiers, l'Etat de droit apparaît plutôt comme un obstacle et non pas comme une garantie de la sécurité publique. Très souvent, ils agissent comme des "gardes frontière ${ }^{38}$, en protégeant les classes dominantes contre les pauvres, cibles préférentielles de la criminalité et de la répression.

D'autres types de violences impliquent des justiciers, des groupes d'extermination, des escadrons de la mort et des tueurs qui s'en prennent à ceux qui sont soupçonnés d'avoir pratiqué des crimes. La violence des gangs qui agissent à la périphérie des principaux centres urbains compte très souvent avec la participation et/ou la complicité de la police militaire ou civile. Des lynchages ont lieu surtout dans des centres urbains comme São Paulo, Rio, Salvador pratiqués par des groupes de personnes incitées à agir illégalement par des victimes de crimes ou par leurs familiers, ou encore, des fois, par des autorités locales (par exemple, des maires, des conseillers municipaux). Dans la plupart des cas, les lynchages ont pour but d'exécuter sommairement des suspects pris en flagrant délit ou extraits de force de leur cellule.

La Constitution de 1988 et la démocratie ont contribué à l'ouverture du pouvoir judiciaire à de nouveaux droits comme celui du consommateur, la protection de l'environnement et la protection plus efficace du droit à la retraite. Il y a même eu des innovations dans le domaine du droit du travail comprenant la reconnaissance des droits des travailleurs domestiques, ce qui a aussi contribué à la création de plusieurs syndicats de cette catégorie professionnelle sur l'ensemble du pays. Cependant, si l'on considère le droit pénal, la situation continue à être très précaire. Les pauvres et les victimes de la criminalité ne perçoivent pas la justice comme une institution de 
protection des droits des classes non privilégiées, mais comme une institution responsable de la criminalisation et de la répression subies par les classes populaires. L'accès des pauvres à la justice est presque inexistant : le nombre de procès élevé, dont la plupart concerne des entreprises, la lenteur des procédures profitant à ceux qui ne veulent pas rembourser leurs dettes ou qui en décalent le paiement, tout ceci porte préjudice à ceux qui ne peuvent se payer un long et coûteux procès ${ }^{39}$.

34 L'application de la loi souffre aussi du racisme. Les accusés noirs ont des peines plus lourdes que celles infligées aux accusés blancs, ce qui indique l'interférence d'un biais racial, comme l'a signalé Sérgio Adorno ${ }^{40}$ à São Paulo. On pourrait dire que l'impunité est virtuellement assurée dans tout le pays pour presque tous ceux qui commettent des crimes contre les « indésirables » ou les « sans pouvoir ». Presque douze années après le massacre de 111 prisonniers dans la Maison d'arrêt Carandiru, dans la ville de São Paulo, aucun parmi la centaine de policiers militaires qui ont participé à ce massacre n'a été condamné... ${ }^{41} \mathrm{Il}$ s'agit d'une situation de justice delayed, justice denied, comme il est dit dans le rapport portant ce titre publié par Amnesty International le 19 mai 2003, et qui constate que finalement au bout de 18 ans, seuls deux propriétaires de fazendas avaient été jugés pour l'assassinat de João Canuto, un leader des travailleurs ruraux. La réforme du pouvoir judiciaire a été décidée après treize années de discussions, espérons que celle-ci permette de limiter les difficultés d'accès à la justice pour tous...

\section{Le gouvernement Cardoso et les droits de l'Homme}

Compte tenu d'une situation de violence montante et de la pratique de graves violations des droits de l'Homme, toutes les administrations fédérales après la fin de la dictature ont pris des initiatives en vue de faire face à ces défis. Le gouvernement du Président Fernando Henrique Cardoso (1995-2002) a poursuivi la politique de ses prédécesseurs en choisissant au cours de ses deux mandats de jouer la carte de la transparence vis-à-vis des violations des droits de l'Homme. Cette politique, qui a été directement définie par le Président lui-même, a pu bénéficier du soutien de quelques uns des membres de son gouvernement, notamment le ministre des Communications et le ministre de la Justice. Certaines critiques qui lui ont été adressées prétendaient qu'une telle politique des droits de l'Homme n'avait pour seul but que d'améliorer l'image du Brésil à l'étranger. En réponse à ces critiques, Cardoso a répondu qu'il ne croyait pas que de simples opérations de communication auraient pu changer l'image du Brésil: celle-ci ne pouvait s'améliorer que si les droits de l'Homme étaient effectivement assurés pour la majorité de la population.

Depuis le retour des civils au gouvernement, le Brésil a signé et ratifié les principaux documents qui composent la charte internationale des droits de l'Homme ${ }^{42}$ comme les pactes des droits économiques, sociaux et culturels et le pacte des droits civils, la convention contre la torture ainsi que, dans le système inter-américain, la convention américaine. Entre 2001 et 2002, le gouvernement a levé les réserves concernant plusieurs traités, avec la ratification de protocoles optionnels permettant que des plaintes individuelles soient directement portées auprès des organismes internationaux. De plus, les rapports soumis à ces organismes ont fait état de manière objective de la situation quelquefois précaire dans laquelle se trouvait le pays du point de vue des dispositions contenues dans ces traités. En 2002, le gouvernement fédéral a décidé d'adresser une invitation ouverte à tous les rapporteurs spéciaux pour les droits 
de l'Homme, pour qu'ils puissent visiter le Brésil sans avoir besoin de demander une autorisation au gouvernement.

La première et plus difficile décision du gouvernement Cardoso fut d'essayer de reconnaître et offrir des réparations relatives aux "personnes disparues suite à leur participation, ou accusation de participation dans des activités politiques dans la période du 2 septembre au 15 août $1979 »^{43}$. Le gouvernement a ainsi fait voter la « loi des disparus » (loi no. 9140, du 4 décembre 1995) ${ }^{44}$. Cette loi a permis la formation d'une commission qui a reconnu la responsabilité de l'Etat concernant ces crimes, a déterminé le montant des indemnités versées aux familles des disparus, a réintégré à leur poste ceux qui avaient bénéficié d'une amnistie politique tout en leur octroyant des compensations financières.

Le gouvernement fédéral a institué un programme national des droits de l'Homme (PNDH I) en $1996^{45}$. La proposition - préparée, sous la coordination du centre d'études de la violence (Núcleo de Estudos da Violência) de l'Université de São Paulo, par des centaines d'organisations des droits de l'Homme au Brésil, à la demande du ministère de la Justice - a réuni plus de 260 suggestions concernant les droits civiques et politiques. Le PNDH I a été fondé sur le principe que le contrôle de la violence endémique exigeait des politiques actives pour faire face à la violence structurelle de la pauvreté, de la faim et du chômage. Par rapport à l'action immédiate, le programme a eu pour objectif le renforcement de l'Etat de droit au Brésil. Quatre ans après son instauration, une partie des propositions du programme a été mise en œuvre, grâce à la planification, la mobilisation et l'organisation de la société civile, dans de nombreux cas, en partenariat avec les agences de l'Etat. Ces décisions prolongeaient des politiques en cours depuis 1990, le gouvernement ayant alors formulé une doctrine selon laquelle la souveraineté nationale ne pouvait servir d'argument contre les accusations de violation des droits de l'Homme. L'Etat brésilien a depuis lors accepté qu'un suivi de la situation des droits de l'Homme dans le pays soit effectué par des organisations de la société civile, nationales et internationales, et a accepté de coopérer avec les systèmes internationaux et inter-américains de protection des droits de l'Homme. En décembre 1998 le Brésil a reconnu la cour inter-américaine des droits de l'Homme.

Après le PNDH d'importants projets de loi ont été adoptés ${ }^{46}$. Parmi les plus importants figure la criminalisation de la torture, la compétence de la juridiction civile pour juger les homicides pratiqués par la police militaire, ainsi qu'un projet de loi ayant pour objectif de faire de la justice fédérale la seule compétente en matière de graves violations des droits de l'Homme (ce projet a été voté fin 2004). Un programme de protection des témoins a également été établi et un premier recensement des fonctionnaires fédéraux tenant compte de critères raciaux et ethniques a été effectué. D'autres programmes ont été élaborés pour faire face à des problèmes cruciaux, comme la recommandation d'un pourcentage minimal d'afro-descendants présents dans les organismes fédéraux. Ont également été institués le programme d'élimination du travail des enfants (PETI), le groupe exécutif d'élimination du travail forcé (GERTRAF) et des groupes de surveillance mobile pour la répression de l'esclavage. Ainsi, pour la première fois, un gouvernement œuvrait pour la mise en place d'une structure permettant de lutter contre ce type de crime.

40 En avril 1997, le gouvernement Cardoso a créé le secrétariat national des droits de l'Homme (devenu secrétariat d'Etat en 1998, le titulaire du portefeuille ayant le rang de ministre $)^{47}$. Un autre signe positif fut le plan national de sécurité publique, lancé en juin 
2002 par le ministre de la justice, José Gregori. Ce plan fut la première proposition d'une politique définie par le gouvernement fédéral pour faire face à la violence, au crime organisé, aux massacres, au blanchiment d'argent, et assurer un contrôle de la circulation des armes à l'échelle nationale. Malheureusement, le Plan n'a pas réussi à imposer des critères clairs de financement du système de sécurité dans les Etats fédérés, ainsi qu'un contrôle plus strict des sommes dépensées. Le PNDH II fut lancé en mai 2002, en prenant en compte les initiatives du PNDH I qui n'avaient pas été réalisées, avec des propositions relatives aux droits économiques, sociaux et culturels. Ce plan était l'expression directe des propositions formulées par des organisations de la société civile.

\section{La société civile : contexte et perceptions}

Comprendre cette période, qui suit le retour à la démocratie dans toute sa complexité, suppose de ne pas laisser de côté le contexte dans lequel le gouvernement opère désormais, en tenant compte surtout des transformations subies par la société civile. Il convient de rappeler que cette situation, marquée par une grande précarité des garanties des droits de l'Homme, a posé des défis énormes aux organisations de la société civile spécialisées... Il s'agit alors de chercher de nouvelles conceptions des droits, et de redéfinir des stratégies en vue de créer des instruments effectifs et viables de protection des droits de l'Homme, en particulier pour les plus démunis. Leurs domaines d'intervention sont en rapport avec des problèmes qu'on pourrait penser hérités d'un siècle révolu : absence d'Etat de droit et non accès à la justice pour ceux qui n'appartiennent pas aux élites; le racisme structurel, et l'impunité dont bénéficie les agents de l'Etat impliqués dans de graves violations des droits de l'Homme.

La société civile contemporaine, soumise à une violence endémique qui s'est répandue depuis la dictature militaire jusqu'à la nouvelle démocratie, impose à l'Etat un défi essentiel : celui du monopole de la violence physique par l'Etat, de la construction d'une sécurité publique c'est-à-dire de la coexistence pacifique des citoyens qui passe par le contrôle de la violence privée. Dans les années 1990, les organisations des droits de l'Homme ont dû faire face à des défis qui dépassaient le simple registre et la dénonciation des violations des droits de la personne, comme cela a été le cas au moment de la résistance à la dictature militaire. La consolidation démocratique exige désormais que de nouveaux liens s'établissent entre les sphères autonomes de la société et les institutions politiques pour qu'un contrôle social s'exerce à l'égard des politiques publiques. Une telle tâche leur a peut-être été facilitée du fait que la promotion des droits de l'Homme est devenue elle-même une politique officielle de gouvernement malgré toutes les limitations que nous avons évoquées pour ce qui concerne leur protection effective. Dès lors, les organisations de la société civile ont pu trouver un cadre de référence pour leur mobilisation. Bien sûr, la situation des groupes les plus vulnérables, comme les peuples indigènes et les populations pauvres dans les grandes régions métropolitaines soumises au contrôle de la terreur des bandes criminelles, reste toujours précaire. Certains territoires, comme les favelas de Rio de Janeiro, pâtissent de l'absence de l'Etat : ainsi, en décembre 2003 deux ministres ont été obligés de négocier avec des narcotrafiquants pour que ces derniers les autorisent à visiter une favela où aurait lieu un spectacle promu par une organisation non gouvernementale. 

perçue comme la "vedette", en opposition à la dictature militaire; et malgré l'existence de ce qu'on pourrait considérer comme un « Etat de non-droit » qui perdure tout au long de la période républicaine, en tout cas pour la majorité pauvre de la population, il convient de noter une intensification et une transformation des luttes des organisations de la société civile depuis les années 1970. Ainsi a perduré la conception d'une société civile en opposition à l'Etat malgré le retour à la constitutionnalité. Dans ce contexte, d'autres conceptions de la société civile ont vu le jour ${ }^{49}: l^{\prime}$ une d'entre elles, qui s'inscrit dans la tradition des Etats-Unis, perçoit la société civile comme complémentaire à l'Etat pour ce qui concerne la gestion des affaires publiques; une autre, qu'on pourrait situer dans une filiation gramscienne, valorise la société civile dans le but d'entreprendre la transformation de l'Etat sans s'engager dans une confrontation avec les appareils d'Etat pour la conquête du pouvoir. En effet, au Brésil la société civile d'aujourd'hui ne s'oppose pas ouvertement à l'Etat (comme elle l'a fait pendant la dictature) mais maintient dans plusieurs situations un partenariat avec l'Etat, gardant son autonomie, en particulier après les années 1990, grâce aussi à plusieurs initiatives de coopération proposées par les gouvernements.

Dès lors, en plus des partenariats engagés avec plusieurs organes de l'administration fédérale, la protection des droits de l'Homme a bénéficié de la création de plusieurs conseils de la citoyenneté ${ }^{50}$. Après 1995, le gouvernement fédéral a commencé à promouvoir un fonctionnement plus effectif de ces organismes comme le conseil de défense des droits de la personne humaine, CDDPH, premier conseil de droits de l'Homme créé dans le pays, dès 1964 par le Président João Goulart et subordonné au ministère de la Justice. Pendant le régime dictatorial, le Conseil n'a pu qu'à de rares moments réaliser ses objectifs. Son but après 1985 est devenu plus concret, puisqu'il s'agissait d'établir la responsabilité de ceux qui portaient atteinte aux droits de l'Homme. Cet engagement a été pris devant la communauté internationale, notamment lors de la ratification des traités de protection des droits de l'Homme dans les années 1990. Ce gouvernement a donné au CDDPH une grande visibilité en analysant des cas exemplaires de violation des droits de l'Homme. Aujourd'hui, la société civile compte également sur de nouveaux instruments créés par l'Etat, comme le médiateur public pour les polices de São Paulo, Belém, Belo Horizonte et Rio de Janeiro, ainsi qu'un ministère public disposant de pouvoirs redéfinis afin de lutter contre les abus de l'Etat, un Janus bifronts qui protège les droits tout en en perpétrant les violations.

Malgré le dynamisme de la société civile dans la protection des droits, on retrouve un niveau très élevé d'acceptation de la violence illégale au sein de la population (sentiment partagé par ailleurs par les élites et par les classes dominantes). Cette attitude accroît les difficultés qui s'interposent à la promotion des droits de l'Homme, et à la lutte contre les formes de violence illégale. De plus, malgré l'existence de mouvements sociaux bien organisés, le principal et quelquefois le seul moyen de participation reste le vote (d'ailleurs obligatoire), la majorité de la population restant en fait à l'écart des organisations de la société civile en dehors des périodes électorales.

Mais malgré cette faible adhésion à la démocratie, la population n'est pas sans percevoir que l'établissement d'un Etat de droit est une exigence vitale pour le gouvernement démocratique. Un sondage d'opinion réalisé par Nancy Cardia, en 1999, dans dix capitales brésiliennes, montre que la société brésilienne préfère la loi aux escadrons de la mort et que la majorité est contre la torture et la brutalité policière ${ }^{51}$. 
Selon cette recherche, la majorité accepte la légitimité des grèves et la protestation sociale pacifique; et ses résultats indiquent aussi que malgré le legs autoritaire du passé récent ou lointain, et malgré les taux encore faibles d'opinions favorables à la démocratie, il existe au sein de la population une sensibilité démocratique.

C'est que la population perçoit clairement l'incapacité des gouvernements démocratiques à assurer la sécurité publique et le bien-être social : cette contradiction apparaît de manière flagrante dans ses attitudes vis-à-vis de la démocratie. Le Brésil est peut-être le pays en Amérique Latine qui a perfectionné le plus les élections et l'accès démocratique aux fonctions publiques. Il est placé en tête parmi les six pays de la région avec le plus haut niveau de participation populaire pendant les élections. Cependant, un sondage récent sur la perception des institutions démocratiques a indiqué que seules $36 \%$ des personnes interrogées se définissaient comme "démocrates", ce qui place le pays au $15^{\text {ème }}$ rang dans un classement où l'Uruguay comprend quant à lui $71,3 \%$ de « démocrates ». Le Brésil se situe ainsi en dessous de la moyenne latino-américaine, qui est de $43 \%$, et $27 \%$ des Brésiliens enquêtés sont allés jusqu'à déclarer "préférer un régime autoritaire pourvu qu'il puisse résoudre les problèmes économiques ", un pourcentage bien plus élevé que la moyenne latino-américaine ${ }^{52}$.

\section{Où en sommes-nous aujourd'hui ?}

Quelle était la situation des droits de l'Homme au Brésil à la fin du gouvernement Fernando Henrique Cardoso ? Si l'on considère le front des droits civiques (parce qu'on ne peut traiter ici de tous les droits) il y a eu des progrès et des reculs et le plus souvent, une stagnation. Dans la lutte pour les droits de l'Homme, le moindre progrès, même très limité, a toujours un impact sur la protection des droits. Il est très difficile d'évoquer en la matière les choses en des termes trop simples, et encore plus de distribuer des notes. Mais l'expérience a été faite: et si je ne peux cacher plus longtemps que j'ai été secrétaire d'Etat aux droits de l'Homme entre le 12 novembre 2001 et le 31 décembre 2002, au cours, par conséquent, de la dernière année du second mandat du Président Fernando Henrique Cardoso, il convient de préciser que j'ai exercé ces fonctions au sein du ministère de la Justice nommé par le Président de la République. J'ai ainsi pu siéger au Conseil des ministres et avoir directement accès au Président. Ma tâche a peut-être été facilitée grâce à ma participation lors de la préparation et mise en place des PNDH I et II. En plus de ces fonctions administratives, comme la présidence de tous les Conseils de protection de droits spécifiques (enfants, afro-descendants, personnes handicapées, etc.), j’ai transféré des responsabilités à la société civile, et ainsi pu faire avancer avec plus d'énergie plusieurs dossiers : travail forcé ou élimination de la torture, exploration sexuelle des enfants. Le secrétaire a pu aussi, avec le ministre de la Justice, suivre des projets de loi ou formuler avec l'accord du ministre, des propositions de loi.

49 Le plus important, je crois, au cours de cette expérience, fût de ne pas renoncer aux interventions en tant que militant des droits de l'Homme: dénoncer les graves violations, lutter contre l'impunité, promouvoir des missions et des visites, interpeller dans chaque Etat de la fédération les autorités responsables de la police civile et militaire. Le premier des trois ministres de la Justice avec lesquels j'ai collaboré, Aloysio Nunes Ferreira, mon ami depuis le temps de son exil à Paris, a proposé au Président une «medida provisória» (décret présidentiel provisoire, dit «mesure» provisoire) qui 
autorisait la police fédérale à prendre en mains les enquêtes dans certains cas de graves violations des droits de l'Homme. Mais dans la plupart des cas, le secrétariat n'avait aucun moyen de peser sur le fonctionnement ou la réforme des agences de l'Etat qui perpétuent des actes graves de violence illégale. Mon approche, le plus souvent, consistait en une opération routinière qui comprenait: la vérification des faits et allégations, la dénonciation par voie de presse, le contact téléphonique avec les ministres concernés et avec les autorités responsables dans les Etats fédérés, la réalisation de missions conjointes avec d'autres collègues du ministère de la Justice, la présentation des cas au CDDPH, l'ouverture les séances du CDDPH à la presse, le partage des initiatives avec le ministre de la Justice. Quelquefois, en tenant compte de la complexité des cas, je prenais contact avec le président de la République : l'accès effectif au président, ou le fait pour certains interlocuteurs de supposer que je pouvais le faire à tout moment, furent des atouts importants.

Pendant tout le temps où je suis resté au gouvernement, je me suis occupé pour l'essentiel des violations de droits de l'Homme perpétrées au niveau des Etats fédérés : tous les matins avant d'arriver à mon bureau, je m'attendais au pire. Heureusement, il n'y a eu qu'un seul cas de torture relevant de la police fédérale, à Rio de Janeiro. J'ai ainsi pu vérifier que les violations sont liées à l'incapacité de l'Etat fédéral à assurer l'application dans chaque Etat des droits garantis par la Convention américaine à l'aide d'instruments qui intègrent la charte des droits de l'Homme au niveau international. Parmi les droits de l'Homme que le Brésil n'est pas capable d'assurer à la majorité de la population figurent le droit à la vie et les droits des victimes. Sans aucune exagération, on peut affirmer que l'Etat brésilien ne contrôle plus les collines de Rio de Janeiro où se trouvent les favelas, ou encore les périphéries de São Paulo, ainsi que les frontières du Centre Ouest et du Nord assiégées par les bandes de narcotrafiquants.

51 Le PNDH a été placé sous la responsabilité du secrétaire d'Etat aux droits de l'Homme. Pour cela il y avait des fonds, très limités d'ailleurs, alloués aux actions de renforcement des droits des noirs, des enfants, des femmes et des handicapés. Des quotas ont été établis pour le recrutement de fonctionnaires noirs dans les administrations fédérales, en particulier dans quelques ministères comme celui de la réforme agraire, de la Justice, et le SEDH. Pour la première fois dans l'histoire brésilienne on a été attentif à l'absence presque complète de noirs dans la carrière diplomatique et on a établi un programme de bourses pour la préparation de candidats afro-descendants. Cependant, mis à part ces initiatives, la plupart des actions prévues dans les deux plans reposaient sur la responsabilité d'autres ministères. Quelques-uns d'entre eux, comme le secrétariat d'Etat à l'action sociale, le ministère du Travail et le ministère de l'Education, ou encore le ministère des Affaires Etrangères ont accepté de coordonner des actions avec le SEDH. La plupart des ministères ne connaissaient pas les PNDH ou ne prêtaient aucune attention à la politique des droits de l'Homme. Nominalement, tous les ministres étaient censés mettre en place le plan, mais très souvent, ils se contentaient d'actions de communication autour des droits de l'Homme, mais sans aucune transcendance sur les actions de leur ministère.

A la fin du gouvernement Cardoso, il restait encore d'énormes obstacles structurels à surmonter afin d'assurer une mise en œuvre effective de la politique des droits de l'Homme. Un pas important n'avait pas été franchi: celui des réformes institutionnelles, comme la réforme de la justice et des polices. Malgré la compétence nouvellement attribuée aux tribunaux civils pour juger des crimes de droit commun 
commis par des policiers militaires, à São Paulo notamment, peut-être à peine un tiers des cas sont, en fait, jugés ${ }^{53}$; et les droits de l'Homme continuent à ne pas être perçus comme un thème important par les hommes politiques. Ceux-ci craignent que l'appui ouvert à la promotion des droits de l'Homme puisse être perçu comme un affaiblissement de leur positionnement «dur » à l'égard de la criminalité. Car la peur du crime continue à être brandie comme un atout majeur dans les campagnes électorales. Plusieurs candidats craignent en effet de s'en prendre ouvertement au crime organisé et de perdre l'appui de groupes d'électeurs issus d'un milieu populaire qui seraient d'éventuels bénéficiaires de ces activités illicites, tout comme, parfois, l'appui financier du crime organisé. Il convient de rappeler également que plusieurs députés fédéraux ont été élus avec l'appui des lobbies des fabricants d'armes et de munitions.

Ainsi, au niveau des droits de l'Homme, persiste le «chiaroscuro » avec un engagement formel qui s'est traduit dans quelques initiatives positives, et malgré des progrès réalisés, comparativement aux débuts de la République, les violations des droits de l'Homme, en particulier des droits civiques, économiques et sociaux persistent. Par moments on s'autorise à croire que malgré les longs délais et les difficultés, la réalisation des droits de l'Homme est engagée sur un chemin de non-retour. Malheureusement, les lenteurs persistent, notamment pour les populations les moins favorisées et cette réalisation dépend trop de la personnalité et de la volonté de ceux qui occupent certaines fonctions au niveau de l'appareil d'Etat. Aussi, nonobstant les engagements internationaux formels pris par le gouvernement brésilien concernant la politique des droits de l'Homme - vu la situation sinistre dans laquelle se débattent les populations pauvres et noires - on ne peut pas encore véritablement parler d'une politique d'Etat.

\section{NOTES}

1. Chef du gouvernement provisoire, 1930-1934 ; président constitutionnel, 1934-1937 ; chef de l'Estado Novo, 1937-1945 ; président constitutionnel, 1950-1954. Il s'est suicidé le 10 août 1954, avant la fin de son mandat. Il n'a eu dans toute l'histoire de l'Amérique Latine que trois présidents qui se sont suicidés : Vargas ; José Manoel Balmaceda du Chili, le 19 septembre 1891 ; et Salvador Allende du Chili, le 11 septembre 1973.

2. Miranda P. de., Comentários à Constituição de 1967,Tomo IV (arts.113-150,\$1), São Paulo, Editora Revista dos Tribunais, 1967, p. 621-713.

3. Singer P., "Movimentos sociais em São Paulo: traços comuns e perspectivas ", in Singer P., Brant V.C., São Paulo: o Povo em Movimento, Petrópolis, Vozes/CEBRAP, 1980, p. 218.

4. José E., Carlos Marighela, São Paulo, Sol e Chuva, 1997, p.194.

5. Debrun, op.cit., p. 16.

6. Newman W.D., The Politics of Aristotle, (4 vol.,), Oxford, 1927, I, p. 223 ; Finley M., L'Invention de la Politique, Paris, Champs/Flammarion, 1985, p. 22. Dans mon argumentation, j'utilise largement et librement les idées de Sir Moses Finley.

7. Frederiksen M.W., Journal of Roman Studies, n57, 1967, p. 254 ; Finley M., op. cit., p. 28. 
8. Selon le recensement de 2001 la population brésilienne de 169369557 comprenait 53,4\% de blancs, 5,6\% de noirs (pretos) et 40,4\% de métis (pardos). Voir IBGE (Insituto Brasileiro de Georgrafia e Estatística), Síntese de Indicadores Sociais 2002, Rio de Janeiro, IBGE, 2003, p. 227.

9. Hall M., Garcia M.A., «Urban Labor », in Connif M.L., McCann F.D., Modern Brazil:Elites and Masses in Historical Perspective, Lincoln et Londres, University of Nebraska Press, 1989, p. 161-191 ; voir aussi Pinheiro P.S. et Hall M.M., A Classe Operária no Brasil, 2 vol., São Paulo, AlfaOmega/Brasiliense,1979-1981.

10. Habermas J. et al., Concversaciones com Herbert Marcuse, Barcelona, Gedisa, 1980, p. 179.

11. Laski H., The State in Theory and Practice, Londres, 1935, p. 57-58 ; Finley M., op. cit., p. 30.

12. Comme le montre Roberto da Matta. Voir en particulier Matta R. da, Carnavals, Bandits, Héros, Paris, Esprit, 1983.

13. Voir le texte magistral de Debrun M., Gramsci: Filosofia, Política e Bom Senso, Canpinas, éditions UNICAMP, 2001.

14. J'utilise ici, bien sûr, le concept de «configuration » de Norbert Elias. Voir, en particulier, Elias N., La Société de Cour, Paris, Flammarion, 1985.

15. Voir Debrun M., A Conciliação e outras Estratégias, São Paulo, Brasiliense, 1983, passim. L'autre référence essentielle est Rodrigues J.H., Conciliação e Reforma no Brasil, Rio de Janeiro, Civilização Brasileira, 1965, 246 p.

16. Comme l'indique C.G. Jung, «l'archétype en soi est vide et purement formel, rien d'autre qu'une facultas performandi, une possibilité de représentation donnée a priori ». Voir Jung C.G. Four Archetypes, Princeton, Princeton University Press, 1992, p. 13. Le rapport établi avec Jung est de ma responsabilité.

17. Debrun M., op. cit., p. 15.

18. Je reprends ici, bien sûr, la catégorie de «trasformismo » créée par Antonio Gramsci. Voir surtout : pour (5), p. 939-940 et pour (36), p. 962, in Gramsci A., Quaderni del Carcere, II, Quaderni 6-11 (1930-1931), Roma, Einaudi, 1977. Voir l'étude historique remarquable de ce phénomène faite par Caroci G., Il Trasformismo dall'Unita'ad Oggi, Milano, Edizione Unicopli, 1992.

19. Comme nous l'a rapellé Keane J., Reflections on Violence, Londres, Verso, 1996, p. 63. Voir aussi Kean J., Civil Society., Oxford, Polity Press, 1998.

20. Brodeur J.P., « Commments on Chevigny », p. 82-83 in Méndez J., O’Donnell G., Pinheiro P.S., (dir.), The (Un)Rule of Law and the Underprivileged in Latin America, Notre Dame, University of Notre Dame Press, 1999 ; pour la version portugaise voir «Comentários sobre Chevigny », p. 100, in Méndez J., O’Donnell G., Pinheiro P.S., (dir.), Democracia, Violência e Pobreza, São Paulo, Paz e Terra, 2000.

21. Poulantzas N., A crise das Ditaduras, Rio de Janeiro, Paz e Terra, 1978, p. 73.

22. Aujourd'hui on peut mentionner le poids des lobbies des policiers militaires, des forces armées contre la reforme des polices ; des entreprises de sécurité privée, dirigées par des anciens policiers ou par des policiers en fonction et de fabricants d'armements et de munitions contre le statut d'armement.Voir aussi Poulantzas N., op. cit., p. 73.

23. Cette préoccupation fut exprimée dans une lettre au président de la République du Brésil par l'Organisation Mondiale contre la Torture et la Fédération Internationale des Droits de l'Homme indiquant que le Brésil a un des plus hauts taux d'assassinat de travailleurs ruraux dans l'Amérique Latine. Voir Chade J., "Assassinatos de sem-terra preocupam estrangeiros », O Estado de S.Paulo, 04 janvier 2005, p. 4.

24. Cette expression a été suggérée pour le contexte des transitions politiques en Europe occidentale par Poulantzas, op. cit., p. 78-79.

25. Ver Cardoso F.H., «Planejamento e política : os anéis burocráticos », A Construção da Demcoracia. Estudos Políticos, Capítulo 5, São Paulo, Siciliano, 1993, p.143-154.

26. Roy A., Le coût de la vie, Paris, Arcades, Gallimard, 1999, p. 40. 
27. Voir l'article de Barros R.P., Henriques R. et Mendonça R., « Desigualdade e Pobreza no Brasil : Retrato de uma Estabilidade Inaceitável », Revista Brasileira de Ciências Sociuais, vol.15, n fevrier 2000, p. 135-136.

28. Dantas F., « Em dez anos, pobreza cai $25 \%$ no país mas cresce nas metrópoles », O Estado de S. Paulo, 26 décembre 2004, p. A4.

29. Kowarick L., «Fatias de Nossa História Recente », in Kowarick L., Escritos Urbanos, Saõ Paulo, Editora 34, 2000, p. 108.

30. «Jovens assassinados », Folha de S. Paulo, 9 juin 2004, p. 2.

31. "Violência no campo bateu recordes ", Jornal do Brasil, 17 avril 2004, p.15. (Commentaire sur le rapport de la Comissão Pastoral da Terra, Conflitos no campo Brasil 2003).

32. Waiselfisz J.J., Mapa da Violência IV, Brasília, UNESCO/Instituto Ayrton Senna/ Secretaria Especial dos Direitos Humanos, 2004, p. 56. Au Brésil les individus déclarent, lors du recensement, la couleur de peau qu'ils entendent être la leur. Par la suite, le recensement dénombre les couleurs indiquées dans trois catégories : noirs (pretos), métis (pardos) et blancs(brancos). Depuis la conférence de l'ONU sur le racisme et la discrimination, Durban, en Afrique du Sud, 2001, les noirs et métis sont désignés dans le langage courant comme des descendants d'Africains (Afrodescendants).

33. Voir « Negro tem menos emprego ; branco ganha 105\% a mais », Folha de S. Paulo, juin 2004, p. A-1.

34. Basaglia F., Ongaro F., Los Crimenes de la Paz, Mexico, Siglo XXI, 1993, p. 157.

35. CEJIL (Centro pela Justiça e o Direito Internacional), Tortura no Brasil. Implementação das Recomendações do Relator da ONU, Rio de Janeiro, CEJIL, 2004, p. 12.

36. Voir Movimento Nacional de Direitos Humanos (MNDH), Relatório Final da Campanha Nacional de Combate à Tortura e à Impunidade, Brasília, MNDH, p. 82-83.

37. Voir Mesquita Neto P., «Aumento de mortes provocadas por policiais ameaça política de segurança pública no Estado de São Paulo », dactylographié, mai 2003. Mesquita informe que toutes les données relatives aux civils tués proviennent de sources officielles fournies par la Corregedoria da Polícia Civil e Corregedoria da Polícia Militar et publiées chaque mois par le Diário Oficial do Estado, la gazette officielle du gouvernement de l'Etat.

38. Comme l'a suggéré Aryeh Neier, qui a employé cette expression lors de sa visite au Brésil en 1987 en tant que directeur de Human Rigths Watch.

39. (Entrevista da 2a. Sergio Renault), «Para secretário, Justiça precisa deixar de ser um 'bom negócio' », Folha de S.Paulo, 27 décembre 2004, p. A14.

40. Adorno S., «Racismo, criminalidade violenta e justiça penal : réus brancos e negros em justiça comparativa », Estudos Históricos, 18, 1996, p. 1-22.

41. Le commandant de l'opération a été condamné à plus de 20 ans de réclusion; selon le droit pénal brésilien, les condamnés peuvent bénéficier, dans un tel cas, d'un nouveau jugement. Entre-temps, il a été élu député, aussi ne peut-il plus être jugé par un jury populaire, mais seulement par des juges professionnels. Jusqu'à cette date, il n'a pas été rejugé.

42. Les instruments suivants font partie de cette Charte : la Déclaration universelle des droits de l'Homme, le Pacte international relatif aux droits économiques, sociaux et culturels, le Pacte international relatif aux droits civils et politiques, le protocole facultatif se rapportant au Pacte international relatif aux droits civils et politiques, le deuxième protocole facultatif se rapportant au Pacte international relatif aux droits civils et politiques visant à abolir la peine de mort.

43. Loi 1940, dite des disparus politiques, décembre 1995.

44. La loi 10.536 du 14 août 2002 a étendu pour une période supplémentaire de neuf ans le délai accordé par la législation précédente, permettant ainsi que des personnes disparues aient leur mort reconnue jusqu'au 5 octobre 1988. 
45. Brésil, Secretaria de Estado dos Direitos Humanos, Direitos Humanos,1995-2002: políticas pblicas de promoção e proteção. (Organizacao de Marcos Vinicius Romano Lemos) Brasilia, Secretaria de Estado dos Direitos Humanos, 2002, p. 9.

46. Ibid., 128 p. ; voir également l'analyse de Adorno S., «Lei e ordem no segundo governo Cardoso », Tempo Social, vol.15, n.2, novembre 2003, p. 103-140.

47. Les titulaires du secrétariat national des droits de l'Homme ont été José Gregori (7 avril 1997 - 14 avril 2000), devenu ensuite en décembre 1998 secrétaire d'Etat, suivi par Gilberto Sabóia (20 juin 2000 - 14 novembre 2001) et Paulo Sérgio Pinheiro (16 octobre 2001 - 31 décembre 2002). En 2003, le gouvernement Lula transforma cet organe en Sécrétariat spécial des droits de l'Homme, attaché à la présidence de la République, son premier titulaire jusqu'à cette date étant Nilmário Miranda.

48. Linz J., Stepan A., Democratic Transition and Consolidation, Baltimore, The John Hopkins University Press, 1996. Voir en particulier 1. «Democracy and its arenas ».

49. Voir Castells M., « The crisis of democracy, global governance, and the rise of the global civil society ", texte présenté dans le cadre du séminaire " Por uma governança global democrática ", Sao Paulo, Instituto Fernando Henrique Cardoso, mai 2004, p. 24-26.

50. Plusieurs conseils de la citoyenneté furent crées après le retour à la démocratie : le Conseil National des Droits de la Femme, CNDM(1988), le Conseil national pour la protection des droits des enfants et des adolescents, CONANDA (1991) et parmi ceux créés par le gouvernement Cardoso, le Conseil national contre la discrimination (1998), le conseil national des droits des handicapés, CONADE (1999), le Conseil national des personnes âgées (2001) et le Conseil national de promotion du droit à l'alimentation, CNPDA (2002) institué après la visite du Rapporteur spécial de l'ONU pour le droit à l'alimentation. Ces conseils comprennent toujours dans leur composition des représentants de la société civile.

51. Cardia N., Primeira pesquisa sobre atitudes, normas culturais e valores em relação à violência em 10 capitais brasilieras.Brasília, Ministério da Justiça/Secretaria de Estado dos Direitos Humanos, 1999

52. «Brasil consolida elições, mas confia pouco na democracia », Prima Pagina, Brasilia, 21 avril 2004, (site www.pnud.org), voir aussi PNUD (Programa de las Naciones Unidas Para el Desarrollo), La Democracia en America Latina. Hacia una democracia de ciudadanos y ciudanas, Buenos Aires, Aguilar, Altea, Taurus, Alfaguara, S.A, 2004.

53. Penteado G., « Ministério Público é conivente com a PM, aponta pesquisa », Folha de S.Paulo, 29 novembre 2004

\section{RÉSUMÉS}

Même dans des moments fondateurs de la démocratisation au Brésil, comme pendant les deux décennies suivant 1985 , les vestiges de l'autoritarisme persistent. Je discute ici les questions relatives aux garanties constitutionnelles, en particulier les droits civils, et le fonctionnement du pouvoir judiciaire et de la police, en essayant d'attirer l'attention sur la violence endémique et les violations systématiques des droits humains sous les gouvernements constitutionnels démocratiques, en particulier des années 1990 à nos jours. J'y examine les efforts déployés au Brésil par le gouvernement et la société civile en vue d'un élargissement de la jouissance des droits de la personne à toute la population. Enfin, cet essai comporte également un témoignage sur mon bref passage au gouvernement fédéral du Brésil. 
Traces of autoritarism remain even in the founding moments - such as the two decades following 1985 - of the Brasilian democracy. I will discuss in this essay aspects related to the constitutional guarantees and in particular civil rights and the functionning of the judiciary power and the police. I will try to spot the light on the endemic violence and on systematical violations of human rights under the democratic constitutionnal governments, and in particular since the 1990s. I will also examine the efforts made by the Brasilian government and civil society aiming at enlarging the full enjoyment of individual rights to the whole population. Finally this essay also includes my personal account on my short stay at the Brasilian federal government.

\section{INDEX}

Index géographique : Brésil

Mots-clés : démocratie, droit

\section{AUTEUR}

\section{PAULO SÉRGIO PINHEIRO}

Paulo Sergio Pinheiro est Visiting Professor of International Relations, à la Brown University, Etats-Unis, et chercheur associé au Centre d'Etudes sur la Violence, université de São Paulo. Il est expert indépendant du secrétaire général des Nations Unies pour l'étude sur la violence contre les enfants. 